\title{
As resoluções de estudantes em situações de proporção simples
}

Tamiles da Silva Oliveira

Eurivalda Ribeiro dos Santos Santana

Adriana Costa Santos da Silva

\section{Resumo}

O objetivo deste é artigo analisar o desempenho e os esquemas de resolução apresentados por estudantes do $3^{\circ}$ e $5^{\circ}$ anos do Ensino Fundamental ao resolverem situações de proporção de simples. O estudo está fundamentado na Teoria dos Campos Conceituais, em específico a Estrutura Multiplicativa. A pesquisa se trata de um recorte dos resultados de um projeto maior, a saber: "Um estudo sobre o domínio das estruturas multiplicativas no Ensino Fundamental" (E-Mult), caracteriza-se por uma pesquisa descritiva e interpretativa, na qual analisamos as resoluções dos estudantes em três situações de proporção simples, que foram resolvidas por 195 estudantes do $3^{\circ}$ ano e 235 estudantes do $5^{\circ}$ ano, de três escolas públicas localizadas no Sul da Bahia. Os resultados apontam que os estudantes de ambos anos escolares apresentam um melhor desempenho na situação a qual se espera o uso da multiplicação, e o desempenho decai quando lidam com as situações nas quais se espera o uso da divisão. Não existem diferenças significativas quando os estudantes lidam com as situações de divisão, os desempenhos de ambos escolares são bem próximo. Os esquemas de resolução que mais levam os estudantes os no $3^{\circ}$ e no $5^{\circ}$ ao acerto das situações é a representação numérica.

Palavras-chave: Proporção simples, Esquemas de resolução, Desempenho. 


\title{
The resolutions of students in situations of proportion simple
}

\author{
Tamiles da Silva Oliveira \\ Eurivalda Ribeiro dos Santos Santana \\ Adriana Costa Santos da Silva
}

\begin{abstract}
\end{abstract}
This article analyzes the performance and scheme of students third and fifth grade high school, when solving mathematical problems of proportion of simple. Specifically, this paper identifies and analyzes the resolution schemes formulated and presented by the students. Therefore, this research is substantiated on the Theory of Conceptual Fields, with emphasis in Multiplicative Structure approach. The study it's a clipping of the research projetc "study on the domain of multiplicative structures in high school", which has a more comprehensive scope of analysis. It was adopted as empirical strategy a descriptive and interpretative research approach. The sample of the survey is composed of 195 students of third grade high school and 235 students fifth grade of three public schools located in the southern region of the state of Bahia, who answered three tests of simple situations. The results show that students from both school years present a better performance in the situation where multiplication is expected, and performance decreases when dealing with the situations in which the use of the division is expected. There are no significant differences when students deal with the situations of division, the performances of both students are very close. The resolution schemes that most lead the students in the 3 rd and $5^{\text {th }}$ to the correctness of situations is the numerical representation.

keyword: Simple proportion, Resolution schemes, performance. 


\section{Introdução}

É sabido que muitos conceitos matemáticos eram utilizados desde a civilizações antigas, e a proporcionalidade é um desses conceitos. Além disso, esse conceito tem aplicabilidade não só na disciplina de matemática, mas também em outras áreas de conhecimento, como, a Física, a Química, a Biologia, entre outras áreas de ensino.

A proporcionalidade é um conteúdo matemático que está presente no cotidiano dos estudantes, no entanto, Nunes (2003) ressalta que na escola ao iniciar o conceito de multiplicação e divisão, não é estabelecida uma relação com o conteúdo de proporção. Apesar desse conceito ser apresentado de maneira formal no $7^{\circ}$ ano do Ensino Fundamental, as diretrizes curriculares salientam que a ideia de proporcionalidade está presente desde os anos inicias, de forma intuitiva por meio de situações que envolvam relações entre grandezas e medidas.

A nossa intenção neste artigo é focar nos desempenhos e esquemas dos estudantes relacionados ao conceito de proporção simples. Intencionando auxiliar intervenções pedagógicas, baseadas nas resoluções explicitados por eles.

O nosso estudo é um recorte de um projeto amplo desenvolvido em rede, intitulado, "Um estudo sobre o domínio das estruturas multiplicativas no Ensino Fundamental" - E-Mult, financiado pela Coordenação de aperfeiçoamento de Pessoal de nível superior CAPES ${ }^{13}$. Esse projeto foi desenvolvido em rede e abrangeu três Estados nordestinos, Bahia (Sede), Pernambuco e Ceará. Cada Estado, tinha quatro escolas parceiras, sendo duas dos anos iniciais do Ensino Fundamental e duas dos anos finais do Ensino Fundamental.

No projeto foi aplicado um instrumento diagnóstico com os estudantes do $1^{\mathrm{o}}$ ao $9^{\mathrm{o}}$ ano do Ensino Fundamental, contendo 13 situações que envolviam conceitos da Estrutura Multiplicativa, no entanto, os sujeitos deste estudo são os estudantes do $3^{\circ}$ e $5^{\circ}$ anos do Ensino Fundamental, dos quais analisaremos a resolução apresentada para três situações de proporção simples do instrumento que foi elaborado.

Com essa análise buscamos responder as seguintes questões de pesquisa: qual o desempenho e os esquemas de resolução apresentados por estudantes do $3^{\circ}$ e $5^{\circ}$ anos do ensino fundamental ao resolverem situações de proporção de simples? Quais as diferenças entre os desempenhos dos estudantes ao final do $3^{\circ}$ e do $5^{\circ}$ anos do ensino fundamental?; Quais as diferenças entre os esquemas de resolução utilizados pelos estudantes ao final do $3^{0}$ e do $5^{\mathrm{o}}$ anos do ensino fundamental? 


\section{Dialogando com uma Teoria Cognitivista}

A teoria dos campos conceituais (TCC) tem o foco na aprendizagem, ela traz contribuições importantes para o processo de ensino da disciplina Matemática, uma vez que permite um diagnóstico da aprendizagem por meio da análise da construção do conhecimento do estudante.

O conhecimento é estruturado em campos conceituais, que se trata de "[...] um conjunto informal e heterogêneo de problemas situações, conceitos, relações, conteúdos, e operações de pensamento, conectados uns aos outros e provavelmente interligados durante o processo de aquisição.” (VERGNAUD 1982, p. 40).

Um campo conceitual envolve diversas situações com níveis de complexidade e contextos distintos. Uma única situação ainda que seja simples, engloba mais de um conceito, por isso, a ideia de um campo conceitual, e não apenas de um conceito. São as diversas situações propostas que irão proporcionar significado ao conceito para o estudante. Alcançar o domínio de um campo conceitual não é uma tarefa simples, é necessário considerar "um longo período de tempo por meio da experiência, maturação e aprendizagem." (VERGNAUD, 1982, p. 40).

Para compreender o processo de desenvolvimento de um conceito é necessário considerar três conjuntos fundamentais, sendo o (S, I, R). O primeiro conjunto é o das situações $(S)$. As situações proporcionam sentido ao conceito, no entanto, o sentido não está na situação por si só, mas, na interação entre o sujeito, a situação e a representação. Todo conceito tem um conjunto de situações com diferentes níveis de complexidade, e para tratar essas situações é necessário mobilizar outros conceitos. Os Invariantes operatórios (I) são o significado, que são as relações e propriedades referentes a um determinado conceito. $\mathrm{O}$ conjunto das representações ( $R$ ), são os significantes que representam as relações e propriedades utilizadas para a resolução de uma situação.

Quando nos referirmos a resolução de situações o conceito de esquema é essencial, pois, são neles que buscamos identificar os conhecimentos envolvidos na ação do sujeito, por meio, das competências que estão sendo apresentadas. Salientamos, que estamos entendendo que as "competências dos estudantes, na resolução de situações-problema, aparecem quando são feitas escolhas corretas." (SANTANA, 2012, p. 45).

Escolhas corretas, no sentido de utilizar invariantes operatórios que são apropriados para a resolução de uma determinada situação, no entanto, as escolhas corretas não garantem a efetivação do esquema. A resolução correta de uma situação está inteiramente ligada a habilidade que o estudante possui. Consideramos, que a habilidade é saber mobilizar os invariantes operatórios escolhidos, de forma que apresentem a resolução correta da tarefa 
solicitada (SANTANA, 2012). Logo, o sucesso em uma tarefa depende de uma combinação entre competência e habilidade.

De acordo com Vergnaud (1990, p.136), “esquema é a organização invariante da conduta para uma dada classe de situações". Compreendemos que o esquema, é a maneira como o estudante irá ordenar suas ações com o objetivo de resolver uma determinada situação ao qual lhe foi proposta. O caráter invariante, é posto de maneira que um esquema pode ser organizado de formas diferenciadas, no entanto, existem elementos que são de fundamental importância para a sua organização e que o indivíduo pode não variar, usando sempre a mesma organização para aquela classe de situações. Quando isso ocorre, afirma-se que esse é o esquema do estudante para aquela classe de situações.

Vergnaud (1990) estudou diferentes campos conceituais, como, o campo conceitual aditivo, o campo conceitual algébrico, no entanto, o foco do nosso estudo está no campo conceitual multiplicativo, que é constituído por situações "cujo tratamento implica uma ou várias multiplicações ou divisões e o conjunto de conceitos e teoremas que permitem analisar estas situações [...]” (VERGNAUD, 1990, p. 8,).

Ainda se destacam nesse campo conceitual, os conceitos de Proporção simples, proporção múltipla, proporção dupla comparação multiplicativa, combinatória e configuração retangular (MAGINA; SANTOS; MERLINI, 2014). Nesse estudo o nosso olhar está voltado para o conceito de proporção simples.

\section{Proporção Simples}

A proporção simples, "trata-se de uma relação quaternária como o próprio nome diz envolve uma relação entre quatro quantidades de naturezas distintas duas a duas, como nos exemplos: pessoas e objetos, bens e custos, tempo e distância, entre outros. ” (MERLINI, 2012, p. 66).

Nas diferentes situações que envolvem esse conceito existem elementos que consideramos pertinentes de serem discutidos de forma detalhada. Podemos destacar os esquemas de resolução (operador escalar e funcional), as operações envolvidas: multiplicação, divisão (partição e quotas) e ainda temos as situações que envolve a classe de um para muitos e muitos para muitos. A seguir passaremos a discutir cada um desses elementos.

Na classe um para muitos, é sempre possível explicitar o valor unitário de uma das quantidades de naturezas distintas. E essa classe está disposta em três categorias de situações, a saber: i) Multiplicação; ii) Divisão por partição e iii) Divisão por cotas. O que diferencia essas três categorias de situações é a posição que a variável desconhecida assume em cada termo da proporção, influenciando no tipo de operação a ser utilizada. (VERGNAUD, 1996). Passaremos 
a discutir sobre cada uma dessas categorias de situações. No Exemplo 1, apresentamos uma situação da categoria multiplicação.

Exemplo 1: Em uma escola há 1o salas de aula. e cada sala de aula é composta por 20 alunos. quantos alunos essa escola tem ao todo?

Ao resolver a situação do Exemplo 1, podemos pensar intuitivamente em resolvê-la como um produto de medida, tratando-a como uma relação ternária: 10 salas de aula $\times 20$ alunos. O que resultaria uma solução correta da situação, no entanto, essa situação não expressa uma relação com apenas três quantidades, e sim uma relação entre quatro quantidades. Para a resolução dessa situação faremos uma análise de um esquema de resolução proposto na TCC utilizando a relação multiplicativa escalar.

Figura 1 - Esquema de resolução utilizando o operador escalar

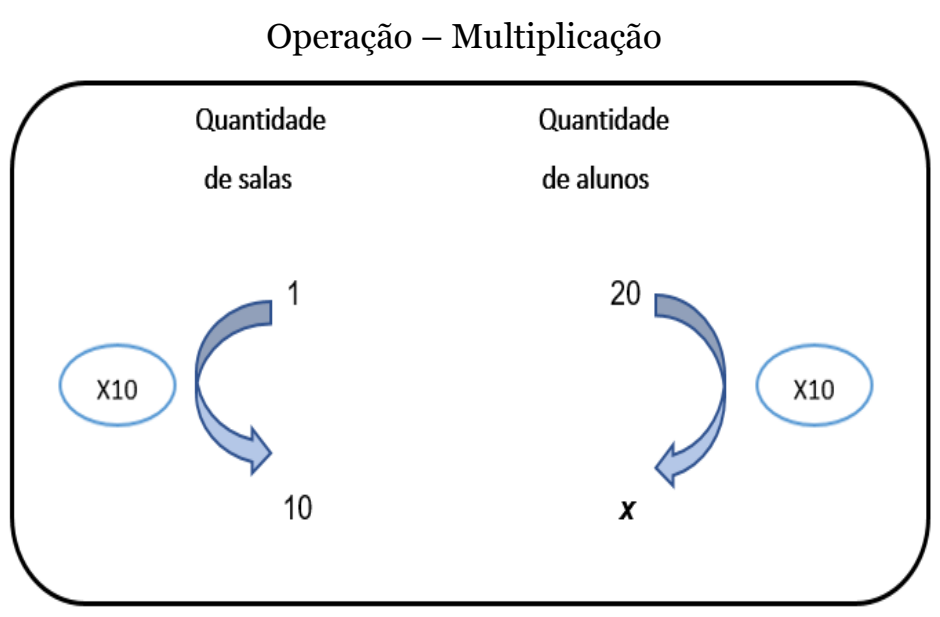

Fonte: Material produzido na pesquisa.

Conforme o esquema apresentado na Figura 1, podemos perceber as relações estabelecidas entre as quatro quantidades e que a correspondência um para muitos está explícita na resolução, na qual temos que a quantidade 1 transforma-se na quantidade 10, por meio do operador escalar $\times 10$. Esse operador escalar não tem dimensão, ele não representa aqui a quantidade de salas ou de alunos, mas se trata de uma replicação, que "envolve somar a cada conjunto a unidade correspondente para o conjunto de modo que a correspondência invariável um-para-muitos seja mantida.” (NUNES, BRYANT, 1997, p. 144). Assim, precisamos de uma relação fixa $\times 10$, para que a proporção seja constante. Inicialmente, descobrimos o operador escalar das quantidades de salas, e usamos o mesmo operador escalar na quantidade de alunos, o que transforma a quantidade 20 em 200 alunos, que é o nosso termo desconhecido $x$. 
Além do operador escalar, temos um outro tipo de operador, o funcional. Apresentamos a seguir na Figura 2 um esquema de resolução envolvendo um operador funcional.

Figura 2 - Esquema de resolução utilizando operador funcional

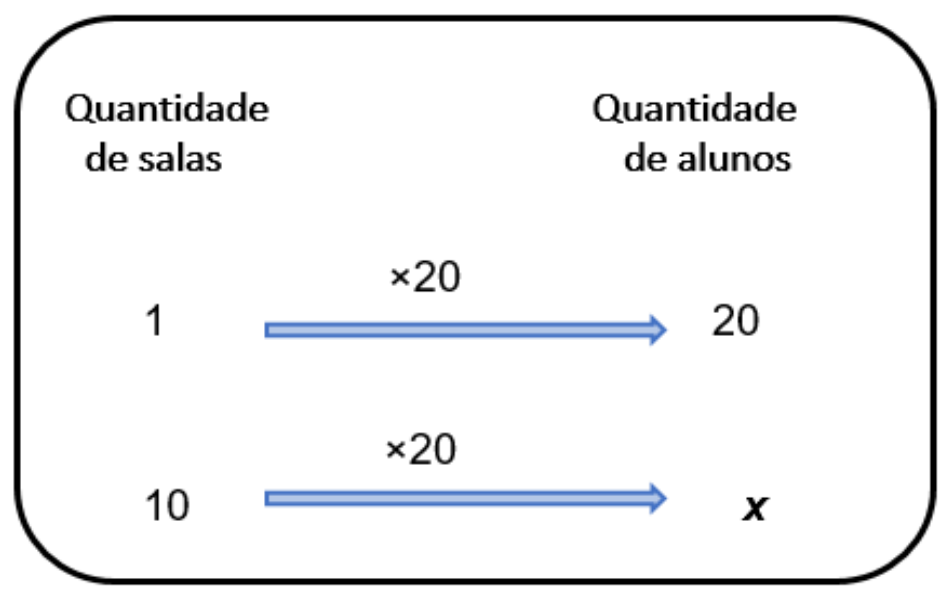

Fonte: Material produzido na pesquisa

É possível observar que no esquema de resolução apresentado na Figura 2, que existe uma relação fixa e constante, diferente da relação estabelecida pelo operador escalar (Figura 1). A relação indicada pela seta horizontal na Figura 2 é um operador funcional $\times 20$. Esse operador representa a relação entre as quantidades. Nesse tipo de representação a proporção simples aparece como um caso especial da função linear observando-se o domínio de validade dessa função. Toda função linear pode ser expressa algebricamente por $\mathrm{f}(x)=\mathrm{ax}$, nesse caso o $a$ é o coeficiente de proporcionalidade, assim temos que:

$\mathrm{f}(1)=\mathrm{a} \times 1$ e f $(10)=\mathrm{a} \times 10$, como o coeficiente é o 20, daí,

$\mathrm{f}(1)=20 \times 1$ sala $=20$ alunos

$\mathrm{f}(10)=20 \times 10$ salas $=200$ alunos

Dessa forma, a função pode ser representada algebricamente por: $\mathrm{f}(x)=20 x$ para $x$ pertencente ao conjunto dos números naturais.

Podemos inferir que a noção intuitiva de função pode ser explorada com os estudantes desde os anos iniciais, a partir das ideias de proporcionalidade. Acreditamos que isso implicará na aprendizagem, pois o conceito de função irá ser explorado formalmente e de maneira aprofundada em anos escolares posteriores.

As situações que envolvem o conceito de proporção simples apresentam níveis de complexidade distintos. Se variarmos a posição do termo desconhecido temos uma diferente ISSN 2526-2882

$$
96 *
$$


complexidade. Apresentamos no Exemplo 2, a seguir, uma situação que envolve a categoria divisão por partição. Nas situações que envolvem essa categoria "é dada uma quantidade inicial e o número de vezes (número de partes) em que esta quantidade deve ser distribuída, devendose encontrar o tamanho de cada parte (número de elementos)". (LAUTERT; SPINILLO, 2002, p. 239).

Exemplo 2: Uma escola composta por 10 salas de aula, tem 200 estudantes. Quantos estudantes tem em cada sala, considerando que cada uma tem a mesma auantidade de estudante?

Apresentamos a seguir na Figura 3 um possível esquema de resolução para o Exemplo 2.

Figura 3 - Esquema de resolução, Operação - Divisão por partição

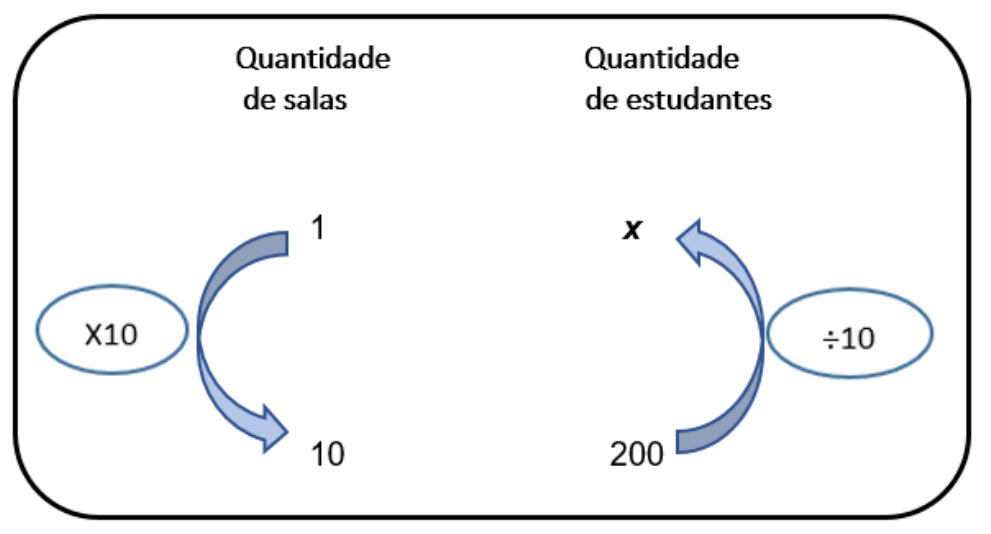

Fonte: Material produzido na pesquisa.

Na Figura 3, temos um esquema de resolução referente a uma situação que envolve a ideia de partição, no qual, precisamos dividir igualmente os 200 estudantes por 10 salas, que vamos encontrar o valor desconhecido 20. Inicialmente dividindo 10 por 1 encontramos o operador escalar $\times 10$, que fez transformar 1 sala em 10 salas. Consequentemente, dividimos 200 estudantes pelo operador escalar 10, pois, essa é operação que possibilita essa transformação. Na divisão partitiva temos que encontrar a quantidade correspondente ao valor unitário e que será possível resolver por meio de uma divisão entre quantidades de natureza distintas.

Além da divisão partitiva, temos um outro tipo de divisão que é a por quotas, o Exemplo 3 explicita uma situação que dá sentido a esse tipo de divisão. Segundo Lautert e Spinillo (2002, p. 238), na "divisão por quotas é dada uma quantidade inicial que deve ser dividida em quotas preestabelecidas (tamanho das partes)”.

Exemplo 3: Uma escola composta por 200 estudantes, sendo que eles ocupam todas as salas de aula. e cada sala de aula é formada apenas por 20 estudanteste. quantas salas de aula essa escola tem? 
Analisamos a resolução do Exemplo 3 na Figura 4.

Figura 4 - Esquema de resolução, Operação - Divisão por quotas

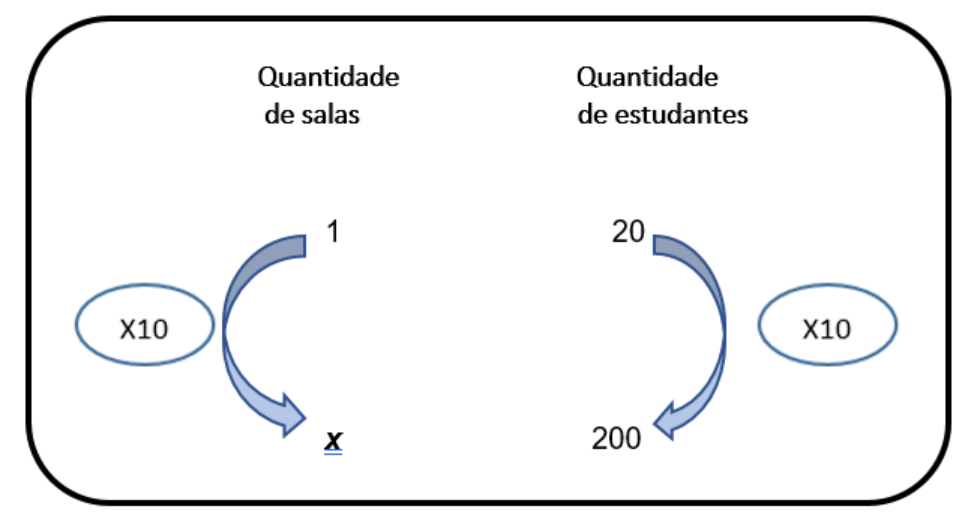

Fonte: Material produzido na pesquisa.

Vejamos que neste esquema de resolução, o objetivo é encontrar as unidades correspondentes ao valor unitário. Temos que encontrar a quantidade de salas suficientes para distribuir a quota de 20 estudantes por sala. identificando quantas quotas de 20 estudantes por sala é possível ter com a quantidade de 200 estudantes. Para resolver essa situação dividimos 200 por 20, e encontramos o operador escalar x 10, assim, para manter a proporção entre as quantidades de naturezas distintas utilizamos o operador escalar $\times 10$ que faz passar de 1 sala para 10 salas, encontrando a solução da situação.

Como podemos perceber ao se tratar da classe de situações de um para muitos, podemos lidar com três categorias de situações, que podem ser resolvidas com multiplicação, com divisão por partição ou com divisão quotas.

\section{Procedimentos Metodológicos}

Este estudo é de abordagem qualitativa de cunho descritivo e interpretativo, pois o método descritivo "tem como objetivo primordial a descrição das características de determinada população ou fenômeno ou, então, o estabelecimento de relações entre variáveis" (GIL, 2002, p.42), o método interpretativo requer "[...] habilidades de observação, comparação, contraste e reflexões que todo humano possui" (ERICKSON, 1986, p. 157) fazendo uso da interpretação que privilegie observação e reflexão de maneira sistemática e discutida.

Sem a interferência do pesquisador no que se refere a promover a aprendizagem do conceito de proporção simples, foi feita uma descrição, interpretação e análise dos resultados encontrados no instrumento diagnóstico, com o intuito de compreender as resoluções registrados pelos estudantes do $3^{\mathrm{o}}$ e $5^{\mathrm{o}}$ anos do ensino fundamental I. 
O universo do estudo são três escolas públicas, localizada no sul da Bahia. Essas escolas foram escolhidas por terem uma parceria com o Grupo de Pesquisa em Educação Matemática Estatística e em Ciências - GPEMEC em ações anteriores, e por atender aos níveis escolares propostos na pesquisa. Participaram do estudo 430 estudantes, sendo 195 do $3^{\circ}$ ano e 235 do $5^{\mathrm{o}}$ ano, com a faixa etária entre 8 a 12 anos.

O foco do estudo é o conceito de proporção simples. Analisamos três situações com níveis de complexidade distintas, distribuídas da seguinte forma: i) Multiplicação, ii) divisão por partição iii) divisão por quotas. Apresentadas no Quadro 1.

Quadro 1 - Situações de proporção simples do instrumento de pesquisa

\begin{tabular}{|l|l|}
\hline \multicolumn{1}{|c|}{ Situação } & Operação esperada para resolução \\
\hline $\begin{array}{l}\text { S1- Joana sabe que em um pacote há 6 biscoitos. } \\
\text { Ela tem 5 pacotes. Quantos biscoitos Joana têm? }\end{array}$ & Multiplicação \\
\hline $\begin{array}{l}\text { S2 - Um supermercadofez uma promoção: “Leve } \\
\text { 4refrigerantes por apenas 12 reais”. Quanto vai } \\
\text { custar cada refrigerante? }\end{array}$ & Divisão por partição \\
\hline $\begin{array}{l}\text { S3 - A Escola Recanto fará uma festa para } 36 \\
\text { convidados. Em cada mesa ficarão } 4 \\
\text { convidados. Quantas mesas a escola precisará } \\
\text { alugar? }\end{array}$ & Divisão quotitiva \\
\hline
\end{tabular}

Fonte: Rede E-Mult (2013-2017).

A análise das resoluções e das respostas dadas pelos estudantes pautou-se em dois blocos:

$\checkmark$ O desempenho dos estudantes.

$\checkmark$ Os esquemas de resolução que levaram os estudantes as respostas certas.

Os esquemas de resolução foram classificados conforme três categorias de análise apresentadas na Figura 5. Essas categorias emergiram da análise dos dados, foram nomeadas e definidas com base na teoria dos campos conceituais. A elaboração das categorias ocorreu no âmbito do projeto de pesquisa E-Mult, num consenso entre os pesquisadores.

Figura 5 - Categorias e subcategorias de classificação dos esquemas de resolução

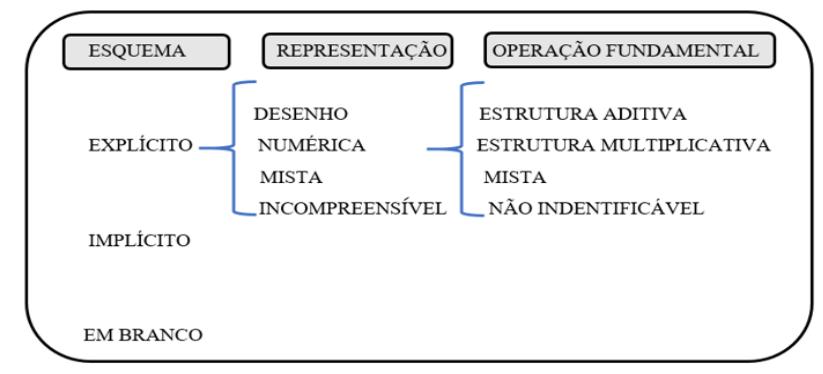

Fonte: Rede E-Mult (2013-2017).

ISSN 2526-2882 
A primeira categoria denominada Esquema, é composta por três subcategorias, a saber: explícito, implícito e em branco. O Quadro 2 apresenta a descrição das subcategorias do Esquema.

Quadro 2- Descrição das subcategorias da categoria Esquema

\begin{tabular}{|l|l|}
\hline Subcategorias do Esquema & Descrição \\
\hline Explícito & $\begin{array}{l}\text { São as ações realizadas pelos estudantes, expressas por meio } \\
\text { de um registro, apresentadas na busca da solução da situação. }\end{array}$ \\
\hline Implícito & $\begin{array}{l}\text { Quando o estudante registra apenas a resposta, sem } \\
\text { apresentar o esquema de resolução utilizado. }\end{array}$ \\
\hline Em branco & $\begin{array}{l}\text { Quando não há nenhum tipo registro no instrumento } \\
\text { diagnóstico. }\end{array}$ \\
\hline
\end{tabular}

Fonte: Material produzido na Rede E-Mult (2013-2017).

As resoluções classificadas como Esquemas explícitos, foram analisadas e classificados nas subcategorias das categorias Representação e Operação Fundamental, descritas no Quadro 3 e 4 respectivamente.

Quadro 3- Descrição das subcategorias Representação

\begin{tabular}{|c|l|}
\hline Representação & \multicolumn{1}{|c|}{ Descrição (Quando o...) } \\
\hline Desenho & $\begin{array}{l}\text { Estudante se utiliza de registros do tipo riscos, traços, bolinhas, dentre outros, } \\
\text { para representar o procedimento de resolução. }\end{array}$ \\
\hline Numérica & Estudante usa apenas números ou símbolos das operações fundamentais. \\
\hline Mista & $\begin{array}{l}\text { Estudante utiliza pelo menos duas das cinco representações (desenho, lista, } \\
\text { diagrama, numérica ou língua materna), sendo representações independentes. }\end{array}$ \\
\hline Incompreensível & $\begin{array}{l}\text { Estudante utiliza uma representação em que não é possível compreender o que } \\
\text { foi registrado. O registro pode ser desenho, número ou outros (letras, rabiscos } \\
\text { não compreensíveis). }\end{array}$ \\
\hline
\end{tabular}

Fonte: Material produzido na Rede E-Mult (2013-2017).

Ao analisar as resoluções observando as operações matemáticas utilizadas, essas foram classificadas segundo a Operação Fundamental registrada pelo estudante, seguindo as subcategorias apresentadas no Quadro 4. 
Quadro 4 - Descrição dos tipos da subcategoria Operação Fundamental

\begin{tabular}{|c|c|}
\hline $\begin{array}{l}\text { Operação } \\
\text { fundamental }\end{array}$ & Descrição \\
\hline Estrutura aditiva & $\begin{array}{l}\text { São os registros relacionadas as operações de adição ou de subtração, quando } \\
\text { o estudante se utiliza das operações ou quando se utiliza das ideias } \\
\text { operacionais das mesmas, podendo ter desenhos atrelados aos símbolos } \\
\text { matemáticos que representam essas operações. }\end{array}$ \\
\hline $\begin{array}{l}\text { Estrutura } \\
\text { multiplicativa }\end{array}$ & $\begin{array}{l}\text { São os registros relacionadas as operações de multiplicação ou de divisão, } \\
\text { quando o estudante se utiliza das operações ou quando se utiliza das ideias } \\
\text { operacionais das mesmas, podendo ter desenhos atrelados aos símbolos } \\
\text { matemáticos que representam essas operações. }\end{array}$ \\
\hline Mista & $\begin{array}{l}\text { Quando o estudante utiliza registros relacionados a uma das operações de } \\
\text { (adição ou subtração) e uma das operaçôes de (Multiplicação ou divisão), ou } \\
\text { quando se utiliza das ideias operacionais das mesmas, podendo ter desenhos } \\
\text { atrelados aos símbolos matemáticos que representam essas operações. }\end{array}$ \\
\hline Não identificável & $\begin{array}{l}\text { Quando não se consegue identificar no registro feito pelo estudante a } \\
\text { operação utilizada. }\end{array}$ \\
\hline
\end{tabular}

Fonte: Material produzido na Rede E-Mult (2013-2017).

As resoluções e respostas registradas pelos estudantes foram analisadas de modo a se responder as questões de pesquisa.

\section{Discutindo Os Dados}

Os dados foram transcritos com a finalidade de analisar as resoluções apresentadas por estudantes do $3^{\circ}$ e $5^{\circ}$ anos do ensino fundamental ao resolverem situações de proporção de simples. Inicialmente consideramos pertinente analisar o desempenho desses estudantes diante das situações que foram propostas. Sendo S1 (multiplicação), S2 (divisão por quotas) e S3 - (divisão por partição). Na Figura 6 apresentamos um gráfico com o desempenho em cada uma dessas situações.

Figura 6 - Desempenho dos estudantes do $3^{\circ}$ e $5^{\circ}$ anos

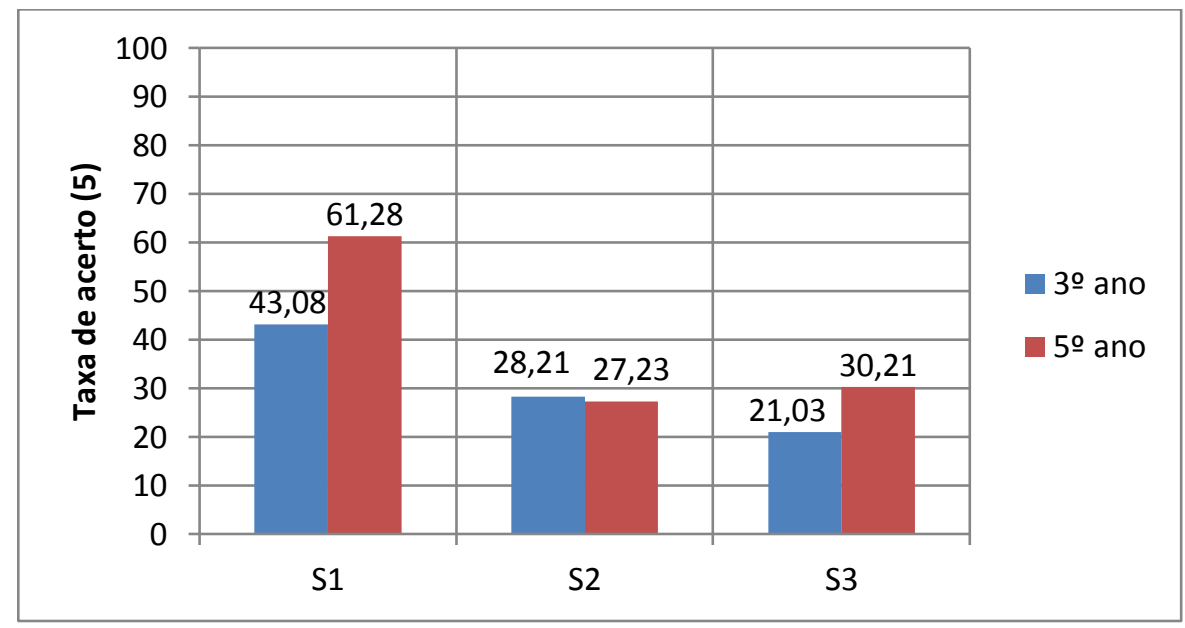

Fonte: Dados da Rede E-Mult (2013-2017).

ISSN 2526-2882 
Podemos observar na Figura 6, o melhor desempenho, em ambos os anos escolares, está concentrado em S1, uma situação na qual se espera o uso da operação de multiplicação em sua resolução. Nas situações $\mathrm{S} 2$ e S3, em que era esperado o uso da operação de divisão para a resolução, o desempenho dos estudantes atinge os mais baixos percentuais de acerto, sendo que os estudantes do $3^{\circ}$ ano obtêm o menor percentual de acerto em $\mathrm{S} 3$ e os estudantes do $5^{\mathrm{o}}$ ano obtêm o menor percentual de acerto em S2. Vejamos na Tabela 1 o resultado do teste quiquadrado.

Com base nos resultados do teste qui-quadrado, a situação $\mathrm{S} 1$ ( $p=0,000)$ é a única situação na qual a diferença de desempenho dos estudantes é estatisticamente significativa. Isto é o desempenhos comparação do desempenho dos estudantes dos dois anos escolares em cada situação, indica que existem diferenças significativas entre os percentuais de acerto. Em S1 e S3 o desempenho dos estudantes do $5^{\circ}$ ano é superior ao do $3^{\circ}$ ano, mas em S2 não é apresentada diferença significativa, ainda que esses estudantes apresentem graus de instrução diferentes como apresentamos na Tabela 1.

Tabela 1 - Taxa de acerto do $3^{\circ}$ e $5^{\circ}$ anos nas situações de proporção simples um para muitos

\begin{tabular}{c|c|c|c|c}
\hline Situação & $\mathbf{3}^{\mathbf{0}}$ ano & $\mathbf{5}^{\mathbf{0}}$ ano & Valor $\boldsymbol{\chi}^{\mathbf{2}}$ & \multicolumn{1}{|c}{ P-valor } \\
\hline S1- Multiplicação & 43,08 & 61,28 & $14,171^{\mathrm{a}}$ &, 000 \\
\hline S2-Divisão quotitiva & 28,21 & 27,23 & $0,050^{\mathrm{a}}$ &, 823 \\
\hline S3-Divisão partitiva & 21,03 & 30,21 & $4,670^{\mathrm{a}}$ &, 031 \\
\hline
\end{tabular}

Fonte: Dados da Rede E-Mult (2013-2017).

Com base nos resultados do teste qui-quadrado, a situação $\mathrm{S} 1(p=0,000)$ é a única situação na qual a diferença de desempenho dos estudantes é estatisticamente significativa. Isto é, o desempenho dos estudantes do $5^{\circ}$ ano apresenta uma diferença relevante em relação ao $3^{0}$ ano. Nas situações $\mathrm{S} 2(p=0,823)$ e S3 $(p=, 031)$ não são apresentadas diferenças significativas, ou seja, ao lidarem com essas situações os estudantes de ambos anos escolares apresentam um desempenho bem próximo.

Ao analisarmos os desempenhos dos estudantes na Situação S1, nos atentamos em classificar dentre as respostas corretas os esquemas explícitos. Estes esquemas foram analisados segundo a representação utilizada pelos estudantes para a resolução, vejamos na Tabela 2 os resultados do $3^{\circ}$ ano. 
Com a Palavra o Professor, Vitória da Conquista (BA), v.3, n. 7, setembro-dezembro / 2018

Tabela 2 - Representação e operação que levam os estudantes do $3^{\circ}$ ano ao acerto S1

\begin{tabular}{l|r|r|r|r|r|r|}
\hline & \multicolumn{7}{|c|}{ Operação } & \\
\cline { 2 - 7 } Representação & $\begin{array}{c}\text { Adição e } \\
\text { Subtração }\end{array}$ & $\begin{array}{c}\text { Multiplicação } \\
\text { e Divisão }\end{array}$ & Mista & $\begin{array}{c}\text { Não } \\
\text { identificado }\end{array}$ & $\begin{array}{c}\text { Não } \\
\text { Classificado }\end{array}$ & \multicolumn{1}{c}{ Total } \\
\hline Desenho & 1,03 & 0,00 & 0,00 & 13,85 & 0,00 & 14,87 \\
\hline Diagrama & 0,00 & 0,51 & 0,00 & 0,00 & 0,00 & 0,51 \\
\hline Numérica & 3,08 & 10,26 & 0,00 & 2,56 & 0,00 & 15,90 \\
\hline Mista & 0,00 & 3,08 & 0,00 & 1,03 & 0,00 & 4,10 \\
\hline Incompreensível & 0,00 & 0,00 & 0,00 & 0,51 & 0,00 & 0,51 \\
\hline Não classificado & 0,00 & 0,00 & 0,00 & 0,00 & 7,18 & 7,18 \\
\hline Total & 4,10 & 13,85 & 0,00 & 17,95 & 7,18 & 43,08 \\
\hline
\end{tabular}

Fonte: Dados da Rede E-Mult (2013-2017).

A Tabela 2 mostra que a representação que mais leva os estudantes do $3^{\circ}$ ano ao acerto é a representação numérica e, a operação mais utilizada não foi identificada pelos pesquisadores e foram feitas com uso de desenhos, das operações identificadas a mais utilizada foi de multiplicação e de divisão. Na Figura 7 apresentamos um esquema de resolução apresentado por um estudante do $3^{\circ}$ ano ao resolver a situação $\mathrm{S} 1$.

Figura 7 - Esquema de resolução apresentado pelo estudante Rafael do $3^{\circ}$ ano

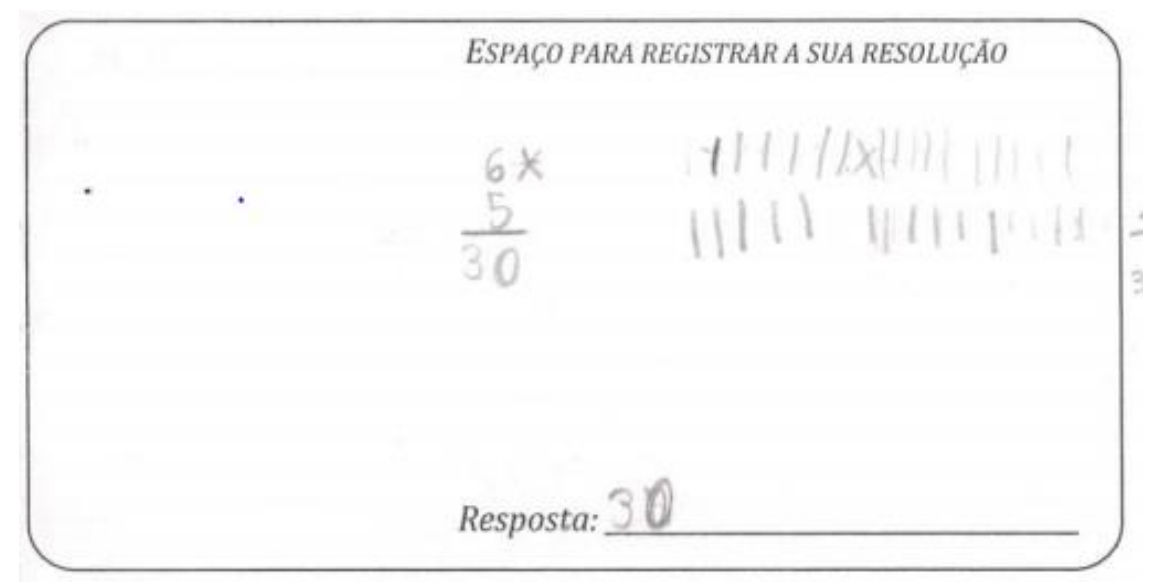

Fonte: Dados da Rede E-Mult (2013-2017).

O estudante se utiliza de uma representação mista, que inclui a numérica e o desenho, para resolver a situação proposta. Apresenta a operação que era esperada para a resolução. Rafael monta o algoritmo com os números 6 X 5 e registra o resultado 30. No entanto, inferimos que existe uma possibilidade do estudante ter registrado os pauzinhos ao lado como auxílio para encontrar o resultado final 30.

A Figura 8 tem um esquema de resolução com a utilização da representação desenho da S2. 
Figura 8 - Esquema de resolução apresentado pela estudante Ana do $3^{\circ}$ ano

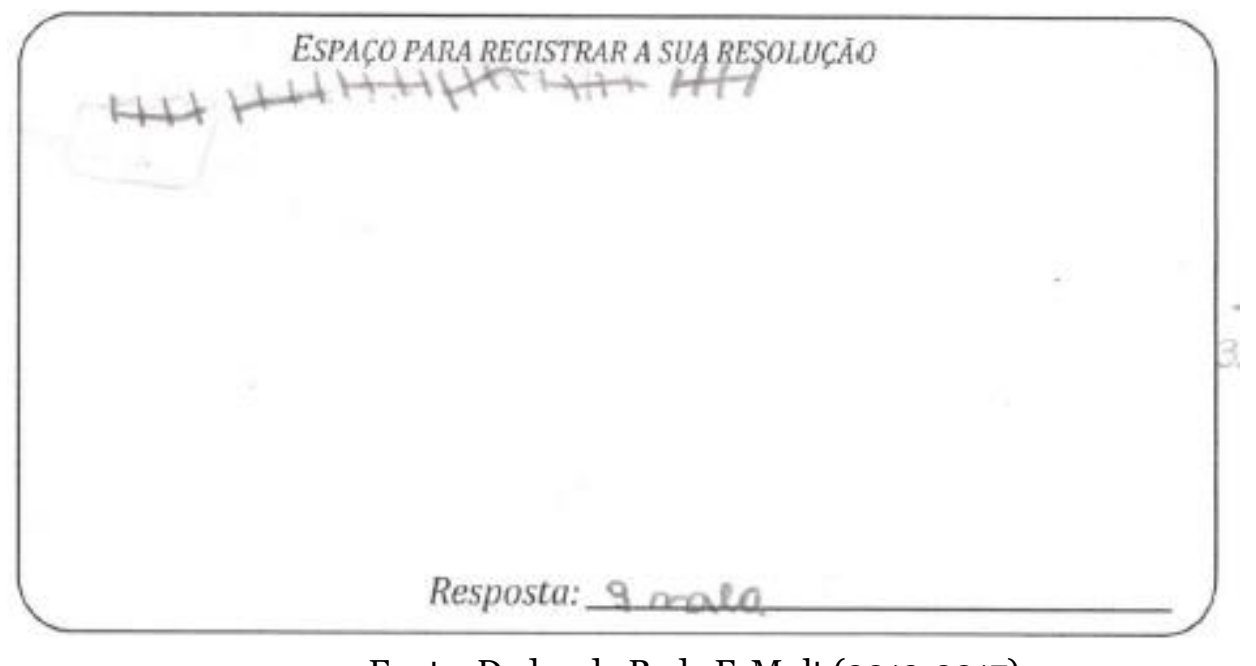

Fonte: Dados da Rede E-Mult (2013-2017).

A estudante Ana apresenta em sua resolução um esquema utilizando desenho, no qual registra agrupamentos de pauzinhos de 4 em 4, entendemos que esses agrupamentos representam a quantidade de convidados em cada mesa. Acreditamos que ela pode ter somado os agrupamentos até chegar em 36 convidados. E chegou a conclusão que para acomodar 36 convidados, são necessários 9 agrupamentos de 4, ou seja, 9 mesas. Diferente do esquema apresentado na Figura 7, este não apresenta o registro de nenhum tipo de algoritmo.

Na Figura 9 apresentamos um exemplo de um esquema que o estudante registra apenas uma representação numérica para a resolução da $S_{3}$.

Figura 9 - Esquema de resolução apresentado pelo estudante Leandro do $3^{\circ}$ ano

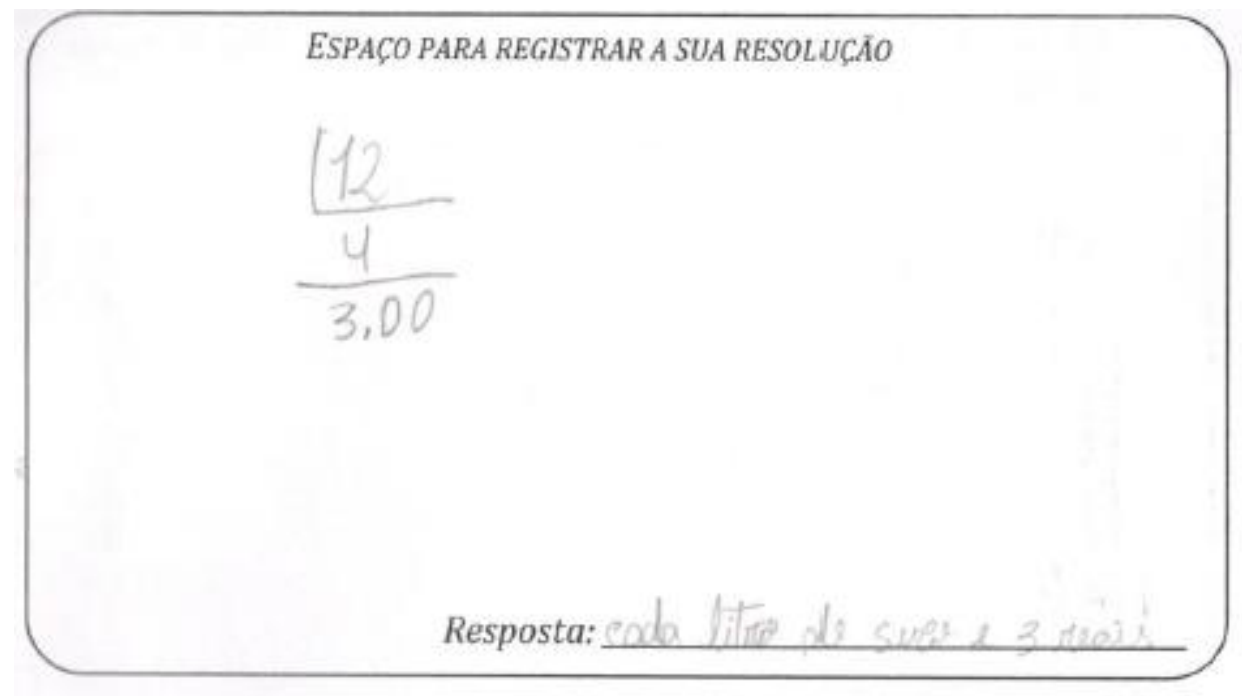

Fonte: Dados da Rede E-Mult (2013-2017). 
Nesse registro inferimos que o estudante Leandro utilizou o algoritmo da divisão. No entanto, ele monta o algoritmo de maneira equivocada, pois o dividendo e o divisor não são apresentadas nas posições que são padronizadas para a operacionalização do algoritmo da divisão. No esquema registrado por Leandro há muitos conhecimentos implícitos e, enquanto pesquisadores, precisaríamos de uma entrevista clínica para identificar as organizações feitas pelo estudante para a resolução.

$\mathrm{Na}$ Tabela 3 apresentamos as representações dos estudantes $5^{\circ}$ ano que levaram ao acerto na situação $\mathrm{S} 1$.

Tabela 3 - Representações que levaram os estudantes do $5^{\circ}$ ano ao acerto

\begin{tabular}{l|r|r|r|r|r|r}
\hline & \multicolumn{7}{|c|}{ Operação } & \multicolumn{1}{c}{} \\
\cline { 2 - 6 } Representação & $\begin{array}{c}\text { Adição e } \\
\text { Subtração }\end{array}$ & $\begin{array}{c}\text { Multiplicação } \\
\text { e Divisão }\end{array}$ & Mista & $\begin{array}{c}\text { Não } \\
\text { identificado }\end{array}$ & $\begin{array}{c}\text { Não } \\
\text { classificado }\end{array}$ & \multicolumn{1}{c}{ Total } \\
\hline Desenho & 0,43 & 0,00 & 0,00 & 4,26 & 0,00 & 4,68 \\
\hline Numérica & 3,40 & 40,85 & 0,43 & 1,70 & 0,00 & 46,38 \\
\hline Mista & 0,00 & 2,13 & 1,28 & 0,85 & 0,00 & 4,26 \\
\hline Incompreensível & 0,00 & 0,00 & 0,00 & 0,43 & 0,00 & 0,43 \\
\hline Não classificado & 0,00 & 0,00 & 0,00 & 0,00 & 5,53 & 5,53 \\
\hline Total & 3,83 & 42,98 & 1,70 & 7,23 & 5,53 & 61,28 \\
\hline
\end{tabular}

Fonte: Dados da Rede E-Mult (2013-2017).

Observamos na Tabela 3 que, como no $3^{\circ}$ ano, a representação mais utilizada pelos estudantes é a numérica. Contudo, a operação mais utilizada foi classificada na subcategoria multiplicação e divisão. E no $5^{\circ}$ ano o uso de desenhos é 69,24\% menos do que no $3^{\circ}$ ano.

Consideramos pertinente analisar a organização que os estudantes do $5^{\circ}$ ano utilizam em seus esquemas de resolução para solucionar as situações propostas. Analisaremos a partir desse momento os esquemas que foram apresentados pelos estudantes do $5^{0}$ ano. Na Figura 10, apresentamos um exemplo de esquemas de resolução referente a S1.

Figura 10 - Esquema de resolução apresentado pelo estudante André do $5^{\circ}$ ano

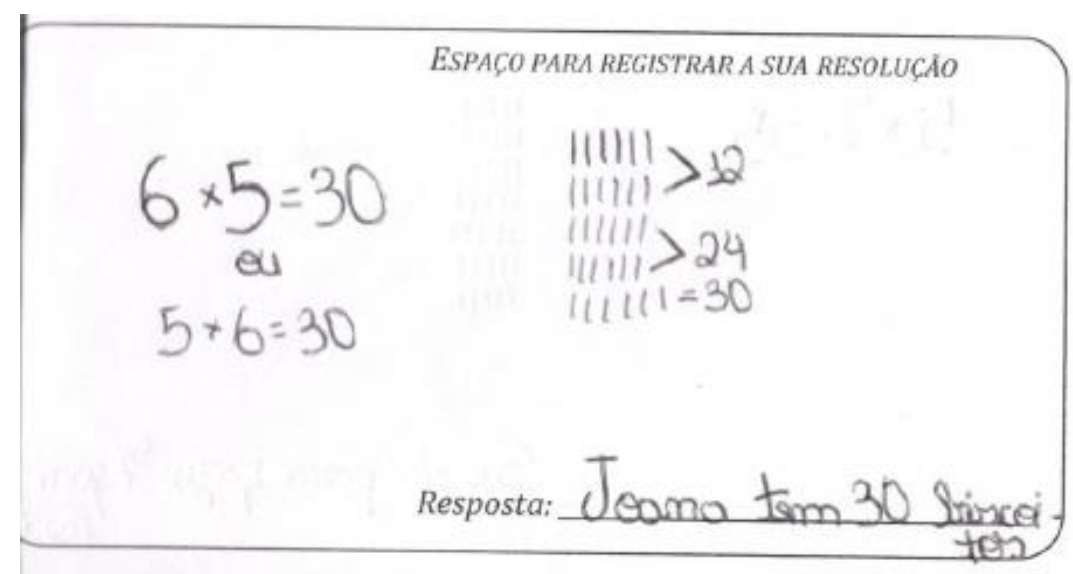

Fonte: Dados da Rede E-Mult (2013-2017).

$$
\text { ISSIN } \angle S \angle 6-\angle Z \square \angle C
$$


O esquema de resolução registrado pelo estudante André se trata de uma representação mista. A resolução apresenta detalhes da sua produção por meio dos conhecimentos explícitos, dos quais nos permite tecer inferências de como o estudante pode ter pensado para organizar o seu esquema. Ele apresenta um agrupamento de pauzinhos de 6 em 6. Em seguida soma os agrupamentos de 12 em 12, como sobrou um agrupamento de seis pauzinhos, junta a última soma com esses, resultando em 30. E, apresenta a multiplicação 6 × $5=30$ ou $5 \times 6=30$, indicando a validade da propriedade comutativa, acreditamos que esse seja um teorema em ação, pois nas escolas brasileiras essa propriedade não é apresentada aos estudantes desse nível escolar. Inferimos que o uso dos dois esquemas, seja utilizado como suporte para encontrar ou para certificar o resultado da multiplicação. Na Figura 11 trazemos um esquema cuja a representação é referente a S3.

Figura 11 - Esquema de resolução apresentado pelo estudante Edu do $5^{\circ}$ ano

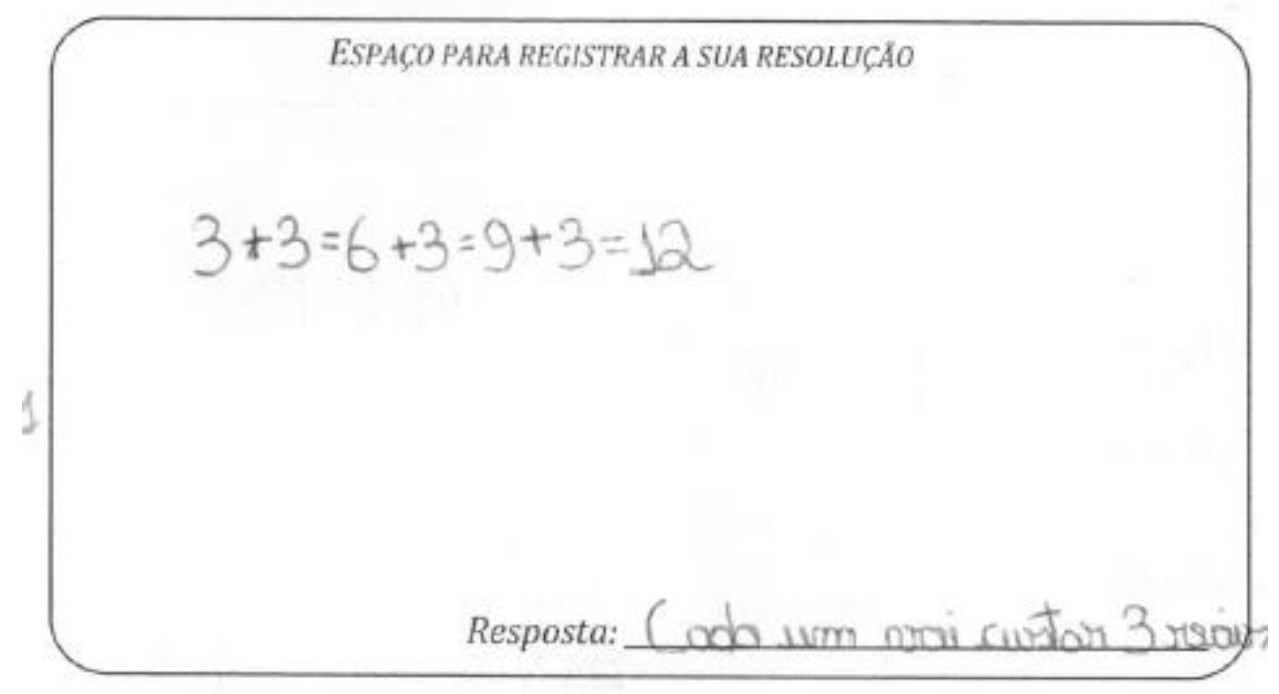

Fonte: Dados da Rede E-Mult (2013-2017).

Nesse esquema o estudante Edu utiliza uma representação numérica. Vemos que ele soma o $3+3$ resultando em 6 . Em seguida soma $6+3$ resultando em 9 e por fim $9+3$ obtendo o resultado 12. É como se ele estivesse fazendo quantas valores de 3,00 reais cabem em 12,00. Contudo, a expressão registrada fica escrita matematicamente errada e, não conseguimos inferir como Edu identificou que o valor unitário seria de 3,oo reais. Para essa resolução uma forma mais simples seria uma divisão por partição de 12 reais dividido igualmente por 4 refrigerantes, que iria resultar em 3,00 reais.

Inferimos que Edu realizou uma operação inversa, buscando por um número em que um total de somado quatro vezes resultasse em 12. Compreendemos que o esquema de resolução apresentado pelo estudante o levou a encontrar o resultado correto da situação, no 
entanto, se a quantidade em dinheiro fosse um número maior que 12, esse tipo de esquema poderia não ser eficaz.

Na Figura 11 o estudante Ivan apresenta um esquema de representação numérica da S3.

Figura 11 - Esquema de resolução apresentado pela estudante Ivan do $5^{\circ}$ ano para $\mathrm{S} 3$

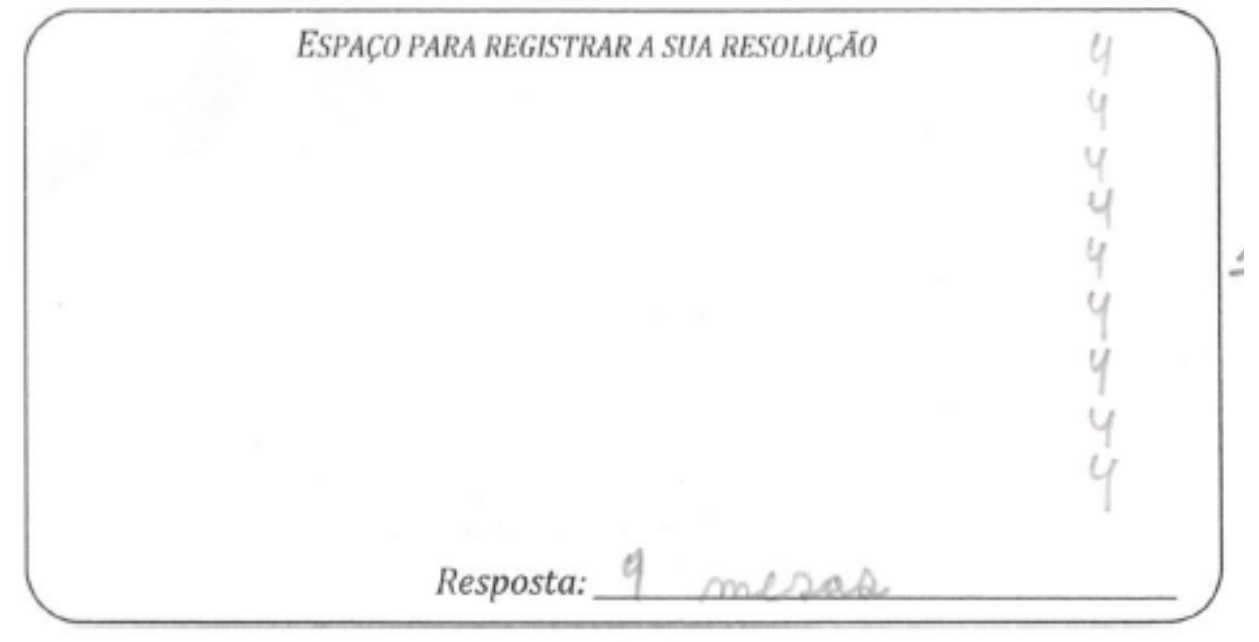

Fonte: Dados da Rede E-Mult (2013-2017).

Observamos, no esquema explícito apresentado na Figura 11, que o estudante Ivan registra nove vezes no número 4 convidados, certamente indicando quatro convidados por mesa, totalizando 36. Edu parece ter buscado identificar quantas cotas de 4 cabem em 36. Ele responde nove mesas. Expressando a resposta correta para $\mathrm{S}_{3}$.

\section{Considerações Finais}

Neste artigo tínhamos as seguintes questões de pesquisa: qual o desempenho e os esquemas de resolução apresentados por estudantes do $3^{\circ}$ e $5^{\circ}$ anos do ensino fundamental ao resolverem situações de proporção de simples? Quais as diferenças entre os desempenhos dos estudantes do $3^{\circ}$ e $5^{\circ}$ anos do ensino fundamental? Quais as diferenças entre os esquemas de resolução utilizados pelos estudantes ao final do $3^{\circ}$ e do $5^{\circ}$ anos do ensino fundamental?

De acordo com a análise dos dados produzidos pudemos constatar que o melhor desempenho dos estudantes em ambos os anos escolares, está concentrado na situação S1, na qual espera-se que mobilizem a operação de multiplicação. Na situação S2 e S3, na qual espera-se que eles mobilizem a operação de divisão, o desempenho deles diminui não apresentam nem 50\% de respostas corretas. Isso significa que eles podem apresentar mais dificuldades quando lidam com a operação de divisão. Esse resultado corrobora com o de Lima (2012), no qual os estudantes revelaram dificuldades na compreensão do conceito de divisão. 
No que se refere as diferenças de desempenhos dos estudantes do $3^{0}$ e $5^{o}$ anos, encontramos diferenças significativas apenas na S1. Na S2 e S3 não detectamos diferenças significativas entre os anos escolares. Apesar dos anos escolares serem diferentes, o desempenho é bem próximo nessas situações. Isto é, independente do ano escolar os estudantes apresentam dificuldades ao lidarem com os conceitos de divisão. Concordamos, com Lima (2012) quando afirma essas dificuldades indicam que possivelmente não vivenciaram situações diversificadas.

Se considerarmos o resultado apresentado como uma reflexão da aprendizagem deles, entendemos que é importante que na sala de aula seja explorada situações nas quais os estudantes precisem mobilizar o conceito de divisão. Pois, é uma operação que esses estudantes ainda não adquiriram domínio. No entanto, entendemos que estes estudantes ainda estão nos anos inicias do ensino fundamental, e precisamos levar em consideração que para alcançar um domínio de um campo conceitual depende de um período de espaço de tempo, que abrange alguns elementos, como a maturação, experiência e a aprendizagem (VERGNAUD, 1982).

Em relação aos esquemas, as representações que mais levam os estudantes ao acerto da situação é a representação numérica em ambos anos escolares, ainda que alguns estudantes utilizem em seus esquemas representações mistas (desenho e numérica), o que prevalece é a representação numérica. Esse resultado pode estar atrelado a forma como esse conceito é trabalhado em sala de aula.

Diante dos resultados apresentados, sugerimos que seja proposto em cursos de formação inicial e continuada que reflexões aos professores da forma como tem sido explorado o conceito de divisão. Proporcionando um ensino que não foque apenas nas formas algorítmica, mas que permitam aos estudantes pensar matematicamente e compreendam os conceitos que estão sendo trabalhado na sala de aula.

\section{Referências}

ERICKSON, F. D. Qualitative methods in research on teaching. In M. C. Wittrock (Ed.), Handbook of research on teaching. 3rd ed. New York, NY: MacMillan, 1986. p. 119161.

Gil, A. Como elaborar projetos de pesquisa. São Paulo: Atlas, 2002.

LAUTERT, S. L.; SPINILLO, A. G. As relações entre o desempenho em problemas de divisão e as concepções de crianças sobre a divisão. Psicologia: Teoria e Pesquisa, Brasília, v. 18, n. 3, 2002. Disponível em: $<$ http://www.scielo.br/scielo.php>. 
MERLINI, V. L. As potencialidades de um processo formativo para a reflexão na e sobre a prática de uma professora nas séries iniciais: Um estudo de caso.Tese (Doutorado em educação matemática).Pontifícia Univesidade Católica de São Paulo. São Paulo, 2012. p.261.

NUNES, T.; BRYANT, P. Criança Fazendo Matemática. Porto Alegre: Artes Médicas, 1997.

SANTANA, E. Estrutura Aditiva: o suporte didático influencia a aprendizagem do estudante? Tese (Doutorado em Educação Matemática) - Pontifícia Universidade Católica de São Paulo. São Paulo, 2010.

VERGnaud. G. A Classification of Cognitive Tasks and Operations of Thought Involved in Addition and Subtraction Problems. In. Addition and Subtraction: a cognitive Perspective. New Jerssey: Lawrense Erlbaun, 1982. P. 3959.

\section{Biografia Resumida}

Tamiles da Silva Oliveira. Graduada em licenciatura em matemática pela universidade Estadual de Santa Cruz (UESC). Mestranda em Educação Matemática pela Universidade Estadual de Santa Cruz (UESC). Membro do grupo de pesquisa em educação matemática, estatística e em ciências (GPEMEC). Tutora externa e professora da educação básica.

Link do Lattes: http://lattes.cnpq.br/3635270256500147

e-mail: tamilesmat@gmail.com

Eurivalda Ribeiro dos Santos Santana. Graduada em Ciências Matemática pela Federação das Escolas Superiores de Ilhéus e Itabuna. Mestre em Matemática pela Universidade Federal da Bahia (UFBA). Doutora em Educação Matemática pela Pontifícia Universidade Católica de São Paulo (PUC/SP). Pós-doutorado em Didática da Matemática pela Universidade de Lisboa. Professora titular na Universidade Estadual de Santa Cruz (UESC/BA). Líder do grupo de pesquisa em educação matemática, estatística e em ciências (GPEMEC).

Link do lattes: http://lattes.cnpq.br/7240586669577145. 
e-mail: eurivalda@uesc.br

Adriana Costa Santos da Silva. Graduada em pedagogia pela Universidade do Estado da Bahia (UNEB). Professora da educação básica.

e-mail: drykka.23@hotmail.com 